Copyright (C) 2009 IEEE This material is presented to ensure timely dissemination of scholarly and technical work. Copyright and all rights therein are retained by authors or by other copyright holders. All persons copying this information are expected to adhere to the terms and constraints invoked by each author's copyright. In most cases, these works may not be reposted without the explicit permission of the copyright holder. 


\title{
On Kalman filtering for 2-D Fornasini-Marchesini models
}

\author{
Ran Yang and Lorenzo Ntogramatzidis and Michael Cantoni
}

\begin{abstract}
This paper deals with the problem of signal estimation for two-dimensional systems. More specifically, we propose a Kalman filter for 2-D systems in Fornasini-Marchesini model, without pre-imposing its structure. It will be shown that the filter thus obtained is not in a Fornasini-Marchesini form, but it still has a recursive structure.
\end{abstract}

\section{INTRODUCTION}

The estimation of the state of a dynamic system from available noisy measurements is a fundamental problem in signal processing, imaging processing and control. The celebrated Kalman filtering approach is by far the most popular estimation approach, as it provides an efficient recursive solution to achieve the minimisation of the covariance of the estimation error [6], [7].

From 1970s, there have been many attempts to extend the Kalman filtering theory to two-dimensional (2-D) systems, see e.g. [5], [8], [11], [15], [16], [19] and the references therein. However, so far the attempts to achieve a truly recursive 2-D Kalman filter were of limited success.

The early works were limited by the difficulty in establishing an effective 2-D recursive latent variable model [5], [8], [15]. In [13], a polynomial solution of 2-D Kalman-Bucy filtering problem was provided. However, the problem thus formulated is less general than the Kalman filter problem, because of its restriction to shift-invariant systems over an infinite horizon.

After the introduction of the Roesser model [12] and Fornasini-Marchesini model [3], in [11], a strip of the semistates of the Roesser model was augmented into a state of 1-D state space model such that 1-D Kalman filter could be applied in 2-D cases. However, it is inherently not 2-D Kalman filter and is hardly applied in practice because of huge dimension of the state variable.

Recently, a recursive Kalman filter for 2-D systems in Fornasini-Marchesini model was developed in [19]. Although the authors claimed that the proposed 2-D Kalman filter minimised the covariance of the estimation error of the state vectors, a Fornasini-Marchersini structure was assumed

This work was supported in part by the Australian Research Council (Discovery Project DP0986577), the National Natural Science Foundation of China (Grant No. 60504022) and the Natural Science Foundation of Guangdong (Grant No. 05003343).

$R$. Yang is with the School of Information Science and Technology, Sun Yat-Sen University, Guangzhou 510275, P.R. China. yangran@mail.sysu.edu.cn

L. Ntogramatzidis is with the Department of Mathematics and Statistics, Curtin University of Technology, Perth WA 6845, Australia. 1.ntogramatzidis@curtin.edu.au

M. Cantoni is with the Department of Electrical and Electronic Engineering, The University of Melbourne, Parkville VIC 3010, Australia. cantoni@unimelb.edu.au on the filter. This implies that the filter thus obtained is not optimal, but optimal among the filters in FornasiniMarchesini form.

In this paper, starting from the formulation of the traditional Kalman filtering problem, a geometric approach is used to derive an estimator without any structure constraint on the filter, so that a truly optimal solution to the minimisation of the variance of estimation error can be achieved. Similarly to the existing 1-D Kalman filter, the proposed 2-D Kalman filter in this paper is also recursive.

Notation. The symbols $\mathbb{C}, \mathbb{Z}$ and $\mathbb{N}$ denote, respectively, the sets of complex, integer and natural numbers (including zero). The symbol $\mathbb{C}^{n}$ denotes the set of complex column vectors with $n$ entries, and $\mathbb{C}^{n \times m}$ represents the set of matrices with complex entries of dimension $n \times m$. The complex conjugate of a matrix $M$ is denoted by $M^{*}$, and $M>0(M \geq 0)$ means that $M$ is positive definite (positive semi-definite). The inequality $(k, l) \leq(i, j)$ is equivalent to $k \leq i$ and $l \leq j$, while $(k, l)<(i, j)$ is equivalent to $(k, l) \leq(i, j)$ but $(k, l) \neq(i, j)$. The symbol $\mathrm{E}\{x\}$ denotes the expected value of a random variable $x$. And $\langle x, y\rangle$ denotes $\mathrm{E}\left\{x y^{*}\right\}$ for column random vectors $x$ and $y$. The symbol $x \perp y$ implies that the zero-mean random variables $x$ and $y$ are uncorrelated. Finally, $\operatorname{span}\left\{w_{1}, w_{2}, \cdots\right\}$ denotes the linear span of the variables $w_{1}, w_{2}, \cdots$.

\section{Problem Formulation}

Consider a linear 2-D system in Fornasini-Marchesini form [3]

$$
\begin{aligned}
x_{i+1, j+1}= & A_{i+1, j}^{(1)} x_{i+1, j}+A_{i, j+1}^{(2)} x_{i, j+1} \\
& +B_{i+1, j}^{(1)} w_{i+1, j}+B_{i, j+1}^{(2)} w_{i, j+1} \\
y_{i, j}= & C_{i, j} x_{i, j}+v_{i, j}
\end{aligned}
$$

where, for all $i, j \in \mathbb{N}, x_{i, j} \in \mathbb{C}^{n}$ and $y_{i, j} \in \mathbb{C}^{p}$ represent the local state and the measurement vector, respectively, while $w_{i, j} \in \mathbb{C}^{m}$ and $v_{i, j} \in \mathbb{C}^{p}$ are the plant (or process) and measurement noises, respectively. Moreover, $A_{i, j}^{(1)}, A_{i, j}^{(2)} \in$ $\mathbb{C}^{n \times n}, B_{i, j}^{(1)}, B_{i, j}^{(2)} \in \mathbb{C}^{n \times m}, C_{i, j} \in \mathbb{C}^{p \times n}$ are known shiftvarying matrices. Let $N_{1}, N_{2} \in \mathbb{N} /\{0\}$. Consider the horizon $\mathcal{S}:=\left\{(i, j) \in \mathbb{Z} \times \mathbb{Z} \mid 0 \leq i \leq N_{1}, 0 \leq j \leq N_{2}\right\}$.

The boundary conditions $x_{i, 0}, x_{0, j}$ and the noises $w_{i, j}$, $v_{i, j}$ are assumed to be zero mean random variables with

$$
\left\langle\left[\begin{array}{c}
w_{i, j} \\
v_{i, j} \\
x_{i, 0} \\
x_{0, j}
\end{array}\right],\left[\begin{array}{c}
w_{k, l} \\
v_{k, l} \\
x_{k, 0} \\
x_{0, l}
\end{array}\right]\right\rangle=\left[\begin{array}{c}
{\left[\begin{array}{cc}
Q_{i, j} & S_{i, j} \\
S_{i, j}^{*} & R_{i, j}
\end{array}\right] \delta_{i k} \delta_{j l}} \\
0 \\
0
\end{array} \quad\left[\begin{array}{cc}
\Pi_{i, 0} \delta_{i k} & 0 \\
0 & \Pi_{0, j} \delta_{j l}
\end{array}\right]\right]
$$


where $\delta_{i k}$ is the 2-D Kronecker delta function

$$
\delta_{i k}= \begin{cases}1 & \text { if } i=k \\ 0 & \text { if } i \neq k\end{cases}
$$

The covariance matrices $Q_{i, j} \in \mathbb{C}^{m \times m}, S_{i, j} \in \mathbb{C}^{m \times p}$, $R_{i, j} \in \mathbb{C}^{p \times p}$ and $\Pi_{i, 0}, \Pi_{0, j} \in \mathbb{C}^{n \times n}$ are known. It is assumed that $R_{i, j}=R_{i, j}^{\top}>0$ for all $i$ and $j$. Similarly to the traditional 1-D Kalman filtering, the 2-D Kalman filtering problem for system (1) can be expressed as follows.

Problem 1: Given the measurements $\left\{y_{k, l} \in \mathbb{C}^{p} \mid 0 \leq\right.$ $(k, l)<(i, j)\}$, find an estimation of $x_{i, j}$, denoted by $\hat{x}_{i, j}$, with $\hat{x}_{k, 0}=0$ and $\hat{x}_{0, l}=0$ for $0 \leq k \leq i$ and $0 \leq l \leq j$, such that the mean square value $\mathrm{E}\left\{\tilde{x}_{i, j} \tilde{x}_{i, j}^{*}\right\}$ is minimised, where $\tilde{x}_{i, j}:=x_{i, j}-\hat{x}_{i, j}$ represents the estimation error.

\section{MAIN RESULTS}

In this section, the Kalman filtering problem for 2-D systems in the form of (1) is investigated. The next lemma gives the expression of the least-mean-square estimator $\hat{x}_{i, j}$ in geometric terms.

Lemma 1: Let $\hat{x}_{i, j}$ be the estimated local state given the measurements $\left\{y_{k, l} \in \mathbb{C}^{p} \mid 0 \leq(k, l)<(i, j)\right\}$, and let $\tilde{x}_{i, j}:=x_{i, j}-\hat{x}_{i, j}$. The local state of the least-mean-square estimator which minimises $\left\langle\tilde{x}_{i, j}, \tilde{x}_{i, j}\right\rangle$, is

$$
\hat{x}_{i, j}=\sum_{(0,0) \leq(k, l)<(i, j)}\left\langle x_{i, j}, e_{k, l}\right\rangle\left\langle e_{k, l}, e_{k, l}\right\rangle^{-1} e_{k, l}
$$

where

$$
e_{k, l}=y_{k, l}-C_{k, l} \hat{x}_{k, l}
$$

is the estimation error.

Proof: The local state $\hat{x}_{i, j}$ of the least-mean-square estimator which minimises $\left\langle\tilde{x}_{i, j}, \tilde{x}_{i, j}\right\rangle$ is given by the projection of $x_{i, j}$ on the subspace $\mathcal{L}_{i, j}:=\operatorname{span}\left\{y_{k, l} \in \mathbb{C}^{p} \mid(0,0) \leq\right.$ $(k, l)<(i, j)\}$.

We want to show that projecting $y_{i, j}$ onto $\mathcal{L}_{i, j}$ leads to the recursive formula

$$
\begin{aligned}
& e_{i, j}=y_{i, j}-\hat{y}_{i, j}, \quad \text { begin with } \\
& e_{i, 0}=y_{i, 0} \quad \text { and } \quad e_{0, j}=y_{0, j}
\end{aligned}
$$

where $\hat{y}_{i, j}$ is the projection of $y_{i, j}$ on the subspace $\mathcal{L}_{i, j}$, i.e., it is the part of the random variable $y_{i, j}$ that is determined by the knowledge of the "past" random variables $\left\{y_{0,0}, y_{0,1}, y_{1,0}, \cdots, y_{i-1, j}, y_{i, j-1}\right\}$. Hence, (6) is true since

$$
\begin{aligned}
& e_{i, 0}=y_{i, 0}-\hat{y}_{i, 0}=y_{i, 0}-C_{i, 0} \hat{x}_{i, 0}=y_{i, 0} \\
& e_{0, j}=y_{0, j}-\hat{y}_{0, j}=y_{0, j}-C_{0, j} \hat{x}_{0, j}=y_{0, j}
\end{aligned}
$$

The remainder is the random variable $e_{i, j}$ which can be regarded as the "innovation" in $y_{i, j}$ given $\left\{y_{0,0}, y_{0,1}, y_{1,0}, \cdots, y_{i-1, j}, y_{i, j-1}\right\}$. Thus $e_{i, j}$ is uncorrelated with $\left\{y_{0,0}, y_{0,1}, y_{1,0}, \cdots, y_{i-1, j}, y_{i, j-1}\right\}$, and then uncorrelated with all the other vectors $\left\{e_{k, l}\right\},(k, l) \neq(i, j)$.

Therefore, vectors $\left\{y_{k, l} \in \mathbb{C}^{p} \mid(0,0) \leq(k, l)<(i, j)\right\}$, which in general are not orthogonal, can be replaced by an equivalent orthogonal set of vectors $\left\{e_{k, l} \in \mathbb{C}^{p} \mid(0,0) \leq\right.$ $(k, l)<(i, j)\}$, in the sense that these two sets of vectors span the same subspace of $\mathbb{C}^{p}$ :

$$
\begin{aligned}
\operatorname{span} & \left\{y_{k, l} \in \mathbb{C}^{p} \mid(0,0) \leq(k, l)<(i, j)\right\} \\
=\operatorname{span} & \left\{e_{k, l} \in \mathbb{C}^{p} \mid(0,0) \leq(k, l)<(i, j)\right\} .
\end{aligned}
$$

This procedure is the well known Gram-Schmidt orthogonalisation procedure, [6].

The orthogonality property of $\left\{e_{k, l} \in \mathbb{C}^{p} \mid(0,0) \leq\right.$ $(k, l)<(i, j)\}$ leads to (3), i.e., the projection of $x_{i, j}$ onto $\mathcal{L}_{i, j}$ is equal to the sum of the separate projection of $x_{i, j}$ onto each of the "previous" orthogonal vectors $e_{k, l},(k, l)<(i, j)$.

Moreover, from (2) we find that $v_{i, j}$ is uncorrelated with the boundary conditions $x_{i, 0}$ and $x_{0, j}$, and is also uncorrelated with $w_{k, l}$ with $(k, l) \neq(i, j)$. It follows that $v_{i, j}$ is uncorrelated with the local state and with the output in the region $\{(k, l) \in \mathbb{N} \times \mathbb{N} \mid(0,0) \leq(k, l)<(i, j)\}$. As such, the projection $\hat{v}_{i, j}$ of $v_{i, j}$ on $\mathcal{L}_{i, j}$ is zero, as it represents the estimate of a white noise. This implies that

$$
\hat{y}_{i, j}=C_{i, j} \hat{x}_{i, j}+\hat{v}_{i, j}=C_{i, j} \hat{x}_{i, j}
$$

Hence (4) holds.

Some orthogonality properties are derived in the following lemma.

Lemma 2: Given a 2-D system in the form of (1) and an estimator of the form (3), for all $i, j \geq 0$

$$
\begin{aligned}
& \text { i) }\left\langle\hat{x}_{i, j}, \tilde{x}_{i, j}\right\rangle=0 \text {; } \\
& \text { ii) }\left\langle x_{i, j}, e_{i, j}\right\rangle=\left\langle x_{i, j}, \tilde{x}_{i, j}\right\rangle C_{i, j}^{*}=\left\langle\tilde{x}_{i, j}, \tilde{x}_{i, j}\right\rangle C_{i, j}^{*} \text {; } \\
& \text { iii) }\left\langle w_{i+1, j}, e_{k, l}\right\rangle=0 \text {, for }(k, l)<(i+1, j+1) \text {; } \\
& \text { iv) }\left\langle w_{i, j+1}, e_{k, l}\right\rangle=0 \text {, for }(k, l)<(i+1, j+1) \text {. }
\end{aligned}
$$

Proof: $i)$ follows from the fact that $\hat{x}_{i, j}$ is the projection of $x_{i, j}$ onto $\mathcal{L}_{i, j}$.

ii) First, notice that $e_{i, j}=y_{i, j}-\hat{y}_{i, j}=C_{i, j} x_{i, j}+$ $v_{i, j}-C_{i, j} \hat{x}_{i, j}=C_{i, j} \tilde{x}_{i, j}+v_{i, j}$. As such, from $\left.i\right)$ we get $\left\langle x_{i, j}, e_{i, j}\right\rangle=\left\langle\hat{x}_{i, j}+\tilde{x}_{i, j}, C_{i, j} \tilde{x}_{i, j}+v_{i, j}\right\rangle=\left\langle\tilde{x}_{i, j}, \tilde{x}_{i, j}\right\rangle C_{i, j}^{*}$. iii) By (1) and (4), both $y_{k, l}$ and $e_{k, l}$ are the linear combinations of the boundary conditions $\left\{x_{s, 0} \in \mathbb{C}^{n}, s \leq k\right\}$, $\left\{x_{0, s} \in \mathbb{C}^{n}, \quad s \leq l\right\}$ and the process noise $\left\{w_{s, t} \in\right.$ $\left.\mathbb{C}^{m}, \quad(s, t)<(k, l)\right\}$. Thus, (2) yields $\left\langle w_{i, j}, e_{k, l}\right\rangle=0$ if $i \geq k$ or $j \geq l$. From $(k, l)<(i+1, j+1)$, we get $k \leq i+1$. Then $\left\langle w_{i+1, j}, e_{k, l}\right\rangle=0$ holds.

iv) can be proved in a similar way to iii), since from $(k, l)<(i+1, j+1)$ we get $l \leq j+1$. Then $\left\langle w_{i, j+1}, e_{k, l}\right\rangle=0$ holds.

Note that the least-mean-square estimator (3) provided in Lemma 1 is far from being computable and applicable. Our aim is now to find the structure of a 2-D filter that achieves the least-mean-square estimation introduced in Lemma 1. The following theorem introduces the 2-D filter that can achieve this goal.

Theorem 1: The 2-D filter with the following form

$$
\begin{aligned}
\hat{x}_{i+1, j+1}= & A_{i+1, j}^{(1)} \hat{x}_{i+1, j}+A_{i, j+1}^{(2)} \hat{x}_{i, j+1} \\
& +\sum_{l=0}^{j} K_{i+1, j+1 ; l}^{(1)} e_{i+1, l}+\sum_{k=0}^{i} K_{i+1, j+1 ; k}^{(2)} e_{k, j+1} \\
e_{i, j}= & y_{i, j}-C_{i, j} \hat{x}_{i, j}
\end{aligned}
$$


with boundary conditions $\hat{x}_{l, 0}=0$ and $\hat{x}_{0, k}=0$ for $0 \leq l \leq$ $j, 0 \leq k \leq i$, minimises

$$
P_{i, j}:=\left\langle\tilde{x}_{i, j}, \tilde{x}_{i, j}\right\rangle=\left\langle x_{i, j}-\hat{x}_{i, j}, x_{i, j}-\hat{x}_{i, j}\right\rangle
$$

where

$$
\begin{aligned}
& K_{i+1, j+1 ; l}^{(1)}\left\{\begin{array}{rl}
\left(A_{i, j+1}^{(2)}\left\langle x_{i, j+1}, \tilde{x}_{i+1, l}\right\rangle C_{i+1, l}^{*}\right. \\
\left.\quad A_{i+1, j}^{(1)} P_{i+1, j} C_{i+1, j}^{*}\right) T_{i+1, l}^{-1} & l=j \\
A_{i, j+1}^{(2)}\left\langle x_{i, j+1}, \tilde{x}_{i+1, l}\right\rangle C_{i+1, l}^{*} T_{i+1, l}^{-1} & l=0, \cdots, j-1
\end{array}\right. \\
& K_{i+1, j+1 ; k}^{(2)} \\
& =\left\{\begin{array}{cl}
\left(A_{i+1, j}^{(1)}\left\langle x_{i+1, j}, \tilde{x}_{k, j+1}\right\rangle C_{k, j+1}^{*}\right. \\
\left.+A_{i, j+1}^{(2)} P_{i, j+1} C_{i, j+1}^{*}\right) T_{k, j+1}^{-1} & k=i ; \\
A_{i+1, j}^{(1)}\left\langle x_{i+1, j}, \tilde{x}_{k, j+1}\right\rangle C_{k, j+1}^{*} T_{k, j+1}^{-1} & k=0, \cdots, i-1 .
\end{array}\right.
\end{aligned}
$$

and

$$
T_{i, j}:=C_{i, j} P_{i, j} C_{i, j}^{*}+R_{i, j}
$$

Proof: In view of Lemma 1, we have

$$
\begin{aligned}
& \hat{x}_{i+1, j+1}=\sum_{(k, l)<(i+1, j+1)}\left\langle x_{i+1, j+1}, e_{k, l}\right\rangle\left\langle e_{k, l}, e_{k, l}\right\rangle^{-1} e_{k, l} \\
& =\sum_{(k, l)<(i+1, j+1)}\left\langle A_{i+1, j}^{(1)} x_{i+1, j}+A_{i, j+1}^{(2)} x_{i, j+1}\right. \\
& \left.\quad+B_{i+1, j}^{(1)} w_{i+1, j}+B_{i, j+1}^{(2)} w_{i, j+1}, e_{k, l}\right\rangle\left\langle e_{k, l}, e_{k, l}\right\rangle^{-1} e_{k, l} \\
& =A_{i+1, j}^{(1)}\left(\sum_{(k, l)<(i+1, j)}\left\langle x_{i+1, j}, e_{k, l}\right\rangle\left\langle e_{k, l}, e_{k, l}\right\rangle^{-1} e_{k, l}\right. \\
& \left.\quad+\sum_{k=0}^{i}\left\langle x_{i+1, j}, e_{k, j+1}\right\rangle\left\langle e_{k, j+1}, e_{k, j+1}\right\rangle^{-1} e_{k, j+1}\right) \\
& \quad+A_{i, j+1}^{(2)}\left(\sum_{(k, l)<(i, j+1)}\left\langle x_{i, j+1}, e_{k, l}\right\rangle\left\langle e_{k, l}, e_{k, l}\right\rangle^{-1} e_{k, l}\right. \\
& \left.\left.\quad+\sum_{l=0}^{j}\left\langle x_{i, j+1}, e_{i+1, l}\right\rangle\left\langle e_{i+1, l}, e_{i+1, l}\right\rangle\right\rangle^{-1} e_{i+1, l}\right) \\
& \quad+A_{i+1, j}^{(1)}\left\langle x_{i+1, j}, e_{i+1, j}\right\rangle\left\langle e_{i+1, j}, e_{i+1, j}\right\rangle^{-1} e_{i+1, j} \\
& \quad+A_{i, j+1}^{(2)}\left\langle x_{i, j+1}, e_{i, j+1}\right\rangle\left\langle e_{i, j+1}, e_{i, j+1}\right\rangle^{-1} e_{i, j+1} \\
& \quad+B_{i+1, j}^{(1)} \sum_{(k, l)<(i+1, j+1)}\left\langle w_{i+1, j}, e_{k, l}\right\rangle\left\langle e_{k, l}, e_{k, l}\right\rangle^{-1} e_{k, l} \\
& \quad+B_{i, j+1}^{(2)} \sum_{(k, l)<(i+1, j+1)}\left\langle w_{i, j+1}, e_{k, l}\right\rangle\left\langle e_{k, l}, e_{k, l}\right\rangle^{-1} e_{k, l}
\end{aligned}
$$

From (3), it is found that

$$
\begin{aligned}
\sum_{(k, l)<(i+1, j)}\left\langle x_{i+1, j}, e_{k, l}\right\rangle\left\langle e_{k, l}, e_{k, l}\right\rangle^{-1} e_{k, l} & =\hat{x}_{i+1, j} \\
\sum_{(k, l)<(i, j+1)}\left\langle x_{i, j+1}, e_{k, l}\right\rangle\left\langle e_{k, l}, e_{k, l}\right\rangle^{-1} e_{k, l} & =\hat{x}_{i, j+1} .
\end{aligned}
$$

In addition, since $e_{i, j}=C_{i, j} \tilde{x}_{i, j}+v_{i, j}$, we find

$$
\left\langle e_{i, j}, e_{i, j}\right\rangle=C_{i, j}\left\langle\tilde{x}_{i, j}, \tilde{x}_{i, j}\right\rangle C_{i, j}^{*}+\left\langle v_{i, j}, v_{i, j}\right\rangle
$$

which leads to (10). Furthermore,

$$
\begin{aligned}
\left\langle x_{i, j+1}, e_{i+1, l}\right\rangle= & \left\langle x_{i, j+1}, \tilde{x}_{i+1, l}\right\rangle C_{i+1, l}^{*}+\left\langle x_{i, j+1}, v_{i+1, l}\right\rangle \\
= & \left\langle x_{i, j+1}, \tilde{x}_{i+1, l}\right\rangle C_{i+1, l}^{*} \\
\left\langle x_{i+1, j}, e_{k, j+1}\right\rangle= & \left\langle x_{i+1, j}, \tilde{x}_{k, j+1}\right\rangle C_{k, j+1}^{*} \\
& +\left\langle x_{i+1, j}, v_{k, j+1}\right\rangle \\
= & \left\langle x_{i+1, j}, \tilde{x}_{k, j+1}\right\rangle C_{k, j+1}^{*}
\end{aligned}
$$

By applying the orthogonality properties in Lemma 2, (11) becomes

$$
\begin{aligned}
\hat{x}_{i+1, j+1} & =A_{i+1, j}^{(1)} \hat{x}_{i+1, j}+A_{i, j+1}^{(2)} \hat{x}_{i, j+1} \\
& +A_{i+1, j}^{(1)} P_{i+1, j} C_{i+1, j}^{*} T_{i+1, j}^{-1} e_{i+1, j} \\
& +A_{i, j+1}^{(2)} P_{i, j+1} C_{i, j+1}^{*} T_{i, j+1}^{-1} e_{i, j+1} \\
& +A_{i+1, j}^{(1)} \sum_{k=0}^{i}\left\langle x_{i+1, j}, \tilde{x}_{k, j+1}\right\rangle C_{k, j+1}^{*} T_{k, j+1}^{-1} e_{k, j+1} \\
& +A_{i, j+1}^{(2)} \sum_{l=0}^{j}\left\langle x_{i, j+1}, \tilde{x}_{i+1, l}\right\rangle C_{i+1, l}^{*} T_{i+1, l}^{-1} e_{i+1, l}
\end{aligned}
$$

The statement follows.

Theorem 1 provides a 2-D Kalman filter with a recursive structure. However, the expressions of the gain matrices $K_{i+1, j+1 ; l}^{(1)}$ and $K_{i+1, j+1 ; k}^{(2)}$ of the filter still involve the terms $\left\langle x_{i, j}, \tilde{x}_{i, j}\right\rangle,\left\langle x_{i, j+1}, \tilde{x}_{i+1, l}\right\rangle$ and $\left\langle x_{i+1, j}, \tilde{x}_{k, j+1}\right\rangle$. Our effort is now devoted to finding expressions for the gains that are suitable for computations.

The next two lemmas are important for solving this problem. In particular, in the next lemma it is shown how the local state vector $x_{i, j}$ can be expressed as a linear combination of the boundary conditions and the process noise.

Lemma 3: For a 2-D system in the form of (1), the state $x_{i, j}$ can be represented by

$x_{i, j}=\sum_{k=1}^{i} \Phi_{i, j ; k, 0} x_{k, 0}+\sum_{l=1}^{j} \Phi_{i, j ; 0, l} x_{0, l}+\sum_{k=0}^{i} \sum_{l=0}^{j} \Psi_{i, j ; k, l} w_{k, l}$

where the matrices $\Phi_{i, j ; k, 0}, \Phi_{i, j ; 0, l} \in \mathbb{C}^{n \times n}$ and $\Psi_{i, j ; k, l} \in$ $\mathbb{C}^{n \times r}$ describe the linear dependence relationships between $x_{i, j}$ and boundary condition $x_{k, 0}, x_{0, l}$ and the plant noise $w_{k, l}$, respectively. The matrices $\Phi_{i, j ; k, 0}, \Phi_{i, j ; 0, l}$ and $\Psi_{i, j ; k, l}$ can be computed recursively by

$$
\begin{aligned}
\Phi_{i, j ; k, 0} & =\sum_{s=1}^{i} F_{i, j ; s, j-1} \cdot \Phi_{s, j-1 ; k, 0} \\
\Phi_{i, j ; 0, l} & = \begin{cases}A_{i-1, j}^{(2)} A_{i-2, j}^{(2)} \cdots A_{0, j}^{(2)}, & l=j ; \\
\sum_{s=1}^{i} F_{i, j ; s, j-1} \cdot \Phi_{s, j-1 ; 0, l}, & l<j\end{cases}
\end{aligned}
$$




$$
= \begin{cases}\Psi_{i, j ; k, l} & k=i, l=j ; \\ B_{i-1, j}^{(2)}, & k=i-1, l=j ; \\ A_{i-1, j}^{(2)} A_{i-2, j}^{(2)} \cdots A_{k+1, j}^{(2)} B_{k, j}^{(2)}, & k<i-1, l=j ; \\ B_{i, j-1}^{(1)}, & k=i, l=j-1 ; \\ A_{i-1, j}^{(2)} A_{i-2, j}^{(2)} \cdots A_{k, j}^{(2)} B_{k, j-1}^{(1)} & \\ +\sum_{s=k}^{i} F_{i, j ; s, j-1} \cdot \Psi_{s, j-1 ; k, j-1}, & k<i, l=j-1 ; \\ \sum_{s=1}^{i} F_{i, j ; s, j-1} \cdot \Psi_{s, j-1 ; k, l}, & l<j-1\end{cases}
$$

where $F_{i, j ; s, j-1} \in \mathbb{C}^{n \times n}$ is given by

$$
F_{i, j ; s, j-1}= \begin{cases}A_{i-1, j}^{(2)} \cdots A_{s, j}^{(2)} A_{s, j-1}^{(1)}, & s=1, \cdots, i-1 ; \\ A_{i, j-1}^{(1)}, & s=i .\end{cases}
$$

with the boundary condition

$$
\begin{aligned}
& \Phi_{s, 0 ; k, 0}=I_{n} \cdot \delta_{s k} ; \\
& \Phi_{0, s ; 0, k}=I_{n} \cdot \delta_{s k} ; \\
& \Psi_{s, 0 ; k, 0}=0 ; \\
& \Psi_{0, s ; 0, k}=0 .
\end{aligned}
$$

Proof: We proceed by induction. The statement is clearly true when $j=1$ in view of the boundary conditions (15). Suppose that for each $x_{s, j-1}, s=1,2, \cdots, i$, there holds

$$
\begin{aligned}
x_{s, j-1}= & \sum_{k=1}^{s} \Phi_{s, j-1 ; k, 0} x_{k, 0}+\sum_{l=1}^{j-1} \Phi_{s, j-1 ; 0, l} x_{0, l} \\
& +\sum_{k=0}^{s} \sum_{l=0}^{j-1} \Psi_{s, j-1 ; k, l} w_{k, l} .
\end{aligned}
$$

It takes only persistence to see that the system model (1) indicates that $x_{i, j}$ is a linear combination of $x_{1, j-1}, x_{2, j-1}$, $\cdots, x_{i, j-1}, x_{0, j}$ and $w_{1, j-1}, w_{2, j-1}, \cdots, w_{i, j-1}, w_{0, j}, w_{1, j}$, $\cdots, w_{i-1, j}$. Indeed,

$$
\begin{aligned}
x_{i, j}= & A_{i, j-1}^{(1)} x_{i, j-1}+A_{i-1, j}^{(2)} x_{i-1, j} \\
& +B_{i, j-1}^{(1)} w_{i, j-1}+B_{i-1, j}^{(2)} w_{i-1, j} \\
= & A_{i, j-1}^{(1)} x_{i, j-1}+A_{i-1, j}^{(2)}\left(A_{i-1, j-1}^{(1)} x_{i-1, j-1}\right. \\
& +A_{i-2, j}^{(2)} x_{i-2, j}+B_{i-1, j-1}^{(1)} w_{i-1, j-1} \\
& \left.+B_{i-2, j}^{(2)} w_{i-2, j}\right)+B_{i, j-1}^{(1)} w_{i, j-1}+B_{i-1, j}^{(2)} w_{i-1, j} \\
= & A_{i, j-1}^{(1)} x_{i, j-1}+A_{i-1, j}^{(2)} A_{i-1, j-1}^{(1)} x_{i-1, j-1} \\
& +B_{i, j-1}^{(1)} w_{i, j-1}+B_{i-1, j}^{(2)} w_{i-1, j} \\
& +A_{i-1, j}^{(2)} B_{i-1, j-1}^{(1)} w_{i-1, j-1}+A_{i-1, j}^{(2)} B_{i-2, j}^{(2)} w_{i-2, j} \\
& +A_{i-1, j}^{(2)} A_{i-2, j}^{(2)} x_{i-2, j} \\
\vdots & \\
= & \left(A_{i-1, j}^{(2)} A_{i-2, j}^{(2)} \cdots A_{0, j}^{(2)}\right) x_{0, j}+A_{i, j-1}^{(1)} x_{i, j-1} \\
& +\sum_{s=1}^{i-1}\left(A_{i-1, j}^{(2)} A_{i-2, j}^{(2)} \cdots A_{i-s, j}^{(2)}\right) A_{i-s, j-1}^{(1)} x_{i-s, j-1}
\end{aligned}
$$$$
\begin{aligned}
\tilde{x}_{i, j}= & \sum_{q=1}^{j} \Theta_{i, j ; 0, q} x_{0, q}+\sum_{p=1}^{i} \Theta_{i, j ; p, 0} x_{p, 0} \\
& +\sum_{p=0}^{i} \sum_{q=0}^{j} \Xi_{i, j ; p, q} w_{p, q}+\sum_{p=0}^{i} \sum_{q=0}^{j} \Pi_{i, j ; p, q} v_{p, q}
\end{aligned}
$$

where

$$
\begin{aligned}
& +B_{i, j-1}^{(1)} w_{i, j-1}+B_{i-1, j}^{(2)} w_{i-1, j} \\
& +\sum_{\substack{i=2 \\
i-1}}^{(2)}\left(A_{i-1, j}^{(2)} A_{i-2, j}^{(2)} \cdots A_{i-s+1, j}^{(2)}\right) B_{i-s, j}^{(2)} w_{i-s, j} \\
& +\sum_{s=1}^{(2)}\left(A_{i-1, j}^{(2)} A_{i-2, j}^{(2)} \cdots A_{i-s, j}^{(2)}\right) B_{i-s, j-1}^{(1)} w_{i-s, j-1} .
\end{aligned}
$$

Replacing all $x_{s, j-1}$ in (17) by (16) leads to (12). This completes the proof.

Lemma 3 provides a recursive scheme for the computation of matrices $\Phi_{i, j ; k, 0}, \Phi_{i, j ; 0, l}$ and $\Psi_{i, j ; k, l}$, given $\Phi_{s, j-1 ; k, 0}$, $\Phi_{s, j-1 ; 0, l}$ and $\Psi_{s, j-1 ; k, l}, s=1,2, \cdots, i$.

Lemma 4: For a 2-D system in the form of (1) and the Kalman filter (7), the estimation error $\tilde{x}_{i, j}$ is a linear combination of boundary condition $x_{k, 0}, x_{0, l}$ and noises $w_{k, l}$ and $v_{k, l}$ for $k \in\{0, \ldots, i\}$ and $l \in\{0, \ldots, j\}$. More precisely,

$$
\begin{aligned}
\Theta_{i, j ; 0, q}= & A_{i, j-1}^{(1)} \Theta_{i, j-1 ; 0, q}+A_{i-1, j}^{(2)} \Theta_{i-1, j ; 0, q} \\
& -\sum_{l=0}^{j-1} K_{i, j ; l}^{(1)} C_{i, l} \Theta_{i, l ; 0, q}-\sum_{k=0}^{i-1} K_{i, j ; k}^{(2)} C_{k, j} \Theta_{k, j ; 0, q} \\
\Theta_{i, j ; p, 0}= & A_{i, j-1}^{(1)} \Theta_{i, j-1 ; p, 0}+A_{i-1, j}^{(2)} \Theta_{i-1, j ; p, 0} \\
& -\sum_{l=0}^{j-1} K_{i, j ; l}^{(1)} C_{i, l} \Theta_{i, l ; p, 0}-\sum_{k=0}^{i-1} K_{i, j ; k}^{(2)} C_{k, j} \Theta_{k, j ; p, 0}
\end{aligned}
$$

$$
\begin{aligned}
& \Xi_{i, j ; p, q} \\
& =\left\{\begin{array}{lr}
0 & q=j, \\
B_{i, j-1}^{(1)} & p=i, q=j-1, \\
B_{i-1, j}^{(2)} & p=i-1, q=j, \\
A_{i, j-1}^{(1)} \Xi_{i, j-1 ; p, q}+A_{i-1, j}^{(2)} \Xi_{i-1, j ; p, q} \\
-\sum_{l=0}^{j-1} K_{i, j ; l}^{(1)} C_{i, l} \Xi_{i, l ; p, q}-\sum_{k=0}^{(2)} K_{i, j ; k}^{(2)} C_{k, j} \Xi_{k, j ; p, q}
\end{array}\right.
\end{aligned}
$$




$$
\begin{aligned}
& \Pi_{i, j ; p, q} \\
& =\left\{\begin{array}{rr}
0, & p=i, q=j, \\
A_{i-1, j}^{(2)} \Pi_{i-1, j ; p, j}-\sum_{k=0}^{i-1} K_{i, j ; k}^{(2)} C_{k, j} \Pi_{k, j ; p, j}-K_{i, j ; p}^{(2)} & q=j, p<i, \\
A_{i, j-1}^{(1)} \Pi_{i, j-1 ; i, q}-\sum_{l=0}^{j-1} K_{i, j ; l}^{(1)} C_{i, l} \Pi_{i, l ; i, q}-K_{i, j ; q}^{(1)} \\
p=i, q<j, \\
A_{i, j-1}^{(1)} \Pi_{i, j-1 ; p, q}+A_{i-1, j}^{(2)} \Pi_{i-1, j ; p, q} \\
-\sum_{l=0}^{j-1} K_{i, j ; l}^{(1)} C_{i, l} \Pi_{i, l ; p, q}-\sum_{k=0}^{i-1} K_{i, j ; k}^{(2)} C_{k, j} \Pi_{k, j ; p, q}, \\
\end{array}\right.
\end{aligned}
$$

with the following boundary conditions

$$
\begin{aligned}
& \Theta_{i, 0 ; p, 0}=I_{n} \cdot \delta_{i p} ; \\
& \Theta_{0, j ; 0, q}=I_{n} \cdot \delta_{j q} ; \\
& \Xi_{i, 0 ; p, 0}=\Xi_{0, j ; 0, q}=0 ; \\
& \Pi_{i, 0 ; p, 0}=\Pi_{0, j ; 0, q}=0 .
\end{aligned}
$$

Proof: By (1) and (7), we can write

$$
\begin{aligned}
\tilde{x}_{i, j}= & x_{i, j}-\hat{x}_{i, j} \\
= & A_{i, j-1}^{(1)} \tilde{x}_{i, j-1}+A_{i-1, j}^{(2)} \tilde{x}_{i-1, j}+B_{i, j-1}^{(1)} w_{i, j-1} \\
& +B_{i-1, j}^{(2)} w_{i-1, j}-\sum_{l=0}^{j-1} K_{i, j ; l}^{(1)}\left(C_{i, l} \tilde{x}_{i, l}+v_{i, l}\right) \\
& -\sum_{k=0}^{i-1} K_{i, j ; k}^{(2)}\left(C_{k, j} \tilde{x}_{k, j}+v_{k, j}\right)
\end{aligned}
$$

Suppose for some $l$ and $k$, there exist matrices $\Theta_{i, l ; 0, q}$, $\Theta_{i, l ; p, 0}, \Xi_{i, l ; p, q}, \Pi_{i, l ; p, q}$ and $\Theta_{k, j ; 0, q}, \Theta_{k, j ; p, 0}, \Xi_{k, j ; p, q}$, $\Pi_{k, j ; p, q}$ such that

$$
\begin{aligned}
\tilde{x}_{i, l}= & \sum_{q=1}^{l} \Theta_{i, l ; 0, q} x_{0, q}+\sum_{p=1}^{i} \Theta_{i, l ; p, 0} x_{p, 0} \\
& +\sum_{p=0}^{i} \sum_{q=0}^{l} \Xi_{i, l ; p, q} w_{p, q}+\sum_{p=0}^{i} \sum_{q=0}^{l} \Pi_{i, l ; p, q} v_{p, q} \\
\tilde{x}_{k, j}= & \sum_{q=1}^{j} \Theta_{k, j ; 0, q} x_{0, q}+\sum_{p=1}^{k} \Theta_{k, j ; p, 0} x_{p, 0} \\
& +\sum_{p=0}^{k} \sum_{q=0}^{j} \Xi_{k, j ; p, q} w_{p, q}+\sum_{p=0}^{k} \sum_{q=0}^{j} \Pi_{k, j ; p, q} v_{p, q}
\end{aligned}
$$

It can be easily proved that for $l=0$ or $k=0$, the boundary condition (22) satisfies the above equations. Replacing $\tilde{x}_{i, l}$ and $\tilde{x}_{k, j}$ in (23), equation (18) follows. This completes the proof.

Remark 1: Note that in (19), (20) and (21),

$$
\begin{array}{ll}
\Theta_{i, l ; 0, q}=0, & \text { for } l<q ; \\
\Theta_{k, j ; p, 0}=0, & \text { for } k<p \\
\Xi_{i, l ; p, q}=0, & \text { for } l \leq q ; \\
\Xi_{k, j ; p, q}=0, & \text { for } k \leq p \\
\Pi_{i, l ; p, q}=0, & \text { for } l \leq q ; \\
\Pi_{k, j ; p, q}=0, & \text { for } k \leq p .
\end{array}
$$

Based on Lemma 3 and 4, an algorithm can be easily developed to compute the two terms $\left\langle x_{i, j+1}, \tilde{x}_{i+1, l}\right\rangle$ and $\left\langle x_{i+1, j}, \tilde{x}_{k, j+1}\right\rangle$ that are required for the computation of the gain matrices of the 2-D Kalman filter.

Theorem 2: For a 2-D system in the form of (1), there hold

$$
\begin{aligned}
\left\langle x_{i, j+1}, \tilde{x}_{i+1, l}\right\rangle= & \sum_{p=1}^{i} \Phi_{i, j+1 ; p, 0} W_{p, 0} \Theta_{i+1, l ; p, 0}^{*} \\
& +\sum_{q=1}^{l} \Phi_{i, j+1 ; 0, q} W_{0, q} \Theta_{i+1, l ; 0, q}^{*} \\
& +\sum_{p=0}^{i} \sum_{q=0}^{l} \Psi_{i, j+1 ; p, q} Q_{p, q} \Xi_{i+1, l ; p, q}^{*} \\
& +\sum_{p=0}^{i} \sum_{q=0}^{l} \Psi_{i, j+1 ; p, q} S_{p, q} \Pi_{i+1, l ; p, q}^{*}
\end{aligned}
$$

and

$$
\begin{aligned}
\left\langle x_{i+1, j}, \tilde{x}_{k, j+1}\right\rangle= & \sum_{p=1}^{k} \Phi_{i+1, j ; p, 0} W_{p, 0} \Theta_{k, j+1 ; p, 0}^{*} \\
& +\sum_{q=1}^{j} \Phi_{i+1, j ; 0, q} W_{0, q} \Theta_{k, j+1 ; 0, q}^{*} \\
& +\sum_{p=0}^{k} \sum_{q=0}^{j} \Psi_{i+1, j ; p, q} Q_{p, q} \Xi_{k, j+1 ; p, q}^{*} \\
& +\sum_{p=0}^{k} \sum_{q=0}^{j} \Psi_{i+1, j ; p, q} S_{p, q} \Pi_{k, j+1 ; p, q}^{*} .
\end{aligned}
$$

Proof: From (12) and (18), it follows that the term $\left\langle x_{i, j+1}, \tilde{x}_{i+1, l}\right\rangle$ can be represented by

$$
\begin{aligned}
\left\langle x_{i, j+1}, \tilde{x}_{i+1, l}\right\rangle & \\
= & \left\langle\sum_{k=1}^{i} \Phi_{i, j+1 ; k, 0} x_{k, 0}+\sum_{l=1}^{j+1} \Phi_{i, j+1 ; 0, l} x_{0, l}\right. \\
& +\sum_{\substack{k=0 \\
i}}^{j+1} \sum_{l=0} \Psi_{i, j+1 ; k, l} w_{k, l}, \sum_{q=1}^{l} \Theta_{i+1, l ; 0, q} x_{0, q} \\
& +\sum_{p=1}^{i+1} \Theta_{i+1, l ; p, 0} x_{p, 0}+\sum_{p=0}^{i+1} \sum_{q=0}^{l} \Xi_{i+1, l ; p, q} w_{p, q} \\
& \left.+\sum_{p=0}^{i+1} \sum_{q=0}^{l} \Pi_{i+1, l ; p, q} v_{p, q}\right\rangle
\end{aligned}
$$


Then (24) follows. Similarly, $\left\langle x_{i+1, j}, \tilde{x}_{k, j+1}\right\rangle$ can be computed as follows

$$
\begin{aligned}
\left\langle x_{i+1, j}, \tilde{x}_{k, j+1}\right\rangle & \\
= & \left\langle\sum_{p=1}^{i+1} \Phi_{i+1, j ; p, 0} x_{p, 0}+\sum_{q=1}^{j} \Phi_{i+1, j ; 0, q} x_{0, q}\right. \\
& +\sum_{p=0}^{i+1} \sum_{q=0}^{j} \Psi_{i+1, j ; p, q} w_{p, q}, \quad \sum_{q=1}^{j+1} \Theta_{k, j+1 ; 0, q} x_{0, q} \\
& +\sum_{p=1}^{k} \Theta_{k, j+1 ; p, 0} x_{p, 0}+\sum_{p=0}^{k} \sum_{q=0}^{j+1} \Xi_{k, j+1 ; p, q} w_{p, q} \\
& \left.+\sum_{p=0}^{k} \sum_{q=0}^{j+1} \Pi_{k, j+1 ; p, q} v_{p, q}\right\rangle .
\end{aligned}
$$

Then (25) follows.

Remark 2: Considering the boundary condition (22), it is easy to see that

$$
\left\langle x_{i, j+1}, \tilde{x}_{i+1,0}\right\rangle=\left\langle x_{i+1, j}, \tilde{x}_{0, j+1}\right\rangle=0
$$

Theorem 2 solves the problem of computing matrices $K_{i, j ; l}^{(1)}$ and $K_{i, j ; k}^{(2)}$ of the Kalman filter (7). The next theorem will give an algorithm for the computation of matrices $P_{i, j}$.

Theorem 3: Matrix $P_{i, j}$ of the Kalman filter (7) can be computed as follows

$$
\begin{aligned}
P_{i, j} & =\left(\sum_{q=1}^{j-1} \Theta_{i, j ; 0, q} W_{0, q} \Phi_{i, j-1 ; 0, q}^{*}\right. \\
& +\sum_{p=1}^{i} \Theta_{i, j ; p, 0} W_{p, 0} \Phi_{i, j-1 ; p, 0}^{*}+\sum_{p=0}^{i} \sum_{q=0}^{j-1} \Xi_{i, j ; p, q} Q_{p, q} \Psi_{i, j-1 ; p, q}^{*} \\
& \left.+\sum_{p=0}^{i} \sum_{q=0}^{j-1} \Pi_{i, j ; p, q} S_{p, q} \Psi_{i, j-1 ; p, q}^{*}\right)\left(A_{i, j-1}^{(1)}\right)^{*} \\
& +\left(\sum_{q=1}^{j} \Theta_{i, j ; 0, q} W_{0, q} \Phi_{i-1, j ; 0, q}^{*}+\sum_{p=1}^{i-1} \Theta_{i, j ; p, 0} W_{p, 0} \Phi_{i-1, j ; p, 0}^{*}\right. \\
& +\sum_{p=0}^{i-1} \sum_{q=0}^{j} \Xi_{i, j ; p, q} Q_{p, q} \Psi_{i-1, j ; p, q}^{*} \\
& \left.+\sum_{p=0}^{i-1} \sum_{q=0}^{j} \Pi_{i, j ; p, q} S_{p, q} \Psi_{i-1, j ; p, q}^{*}\right)\left(A_{i-1, j}^{(2)}\right)^{*} \\
& +B_{i, j-1}^{(1)} Q_{i, j-1}\left(B_{i, j-1}^{(1)}\right)^{*}+B_{i-1, j}^{(2)} Q_{i-1, j}\left(B_{i-1, j}^{(2)}\right)^{*} .
\end{aligned}
$$

Proof: From (8) and the structure of the 2-D system (1), it is found that

$$
\begin{aligned}
P_{i, j}= & \left\langle\tilde{x}_{i, j}, \tilde{x}_{i, j}\right\rangle=\left\langle\tilde{x}_{i, j}, x_{i, j}\right\rangle \\
= & \left\langle\tilde{x}_{i, j}, A_{i, j-1}^{(1)} x_{i, j-1}+A_{i-1, j}^{(2)} x_{i-1, j}\right. \\
& \left.+B_{i, j-1}^{(1)} w_{i, j-1}+B_{i-1, j}^{(2)} w_{i-1, j}\right\rangle
\end{aligned}
$$

where

$$
\begin{aligned}
& \left\langle\tilde{x}_{i, j}, x_{i, j-1}\right\rangle= \\
& =\sum_{q=1}^{j-1} \Theta_{i, j ; 0, q} W_{0, q} \Phi_{i, j-1 ; 0, q}^{*}+\sum_{p=1}^{i} \Theta_{i, j ; p, 0} W_{p, 0} \Phi_{i, j-1 ; p, 0}^{*} \\
& +\sum_{p=0}^{i} \sum_{q=0}^{j-1}\left(\Xi_{i, j ; p, q} Q_{p, q}+\Pi_{i, j ; p, q} S_{p, q}\right) \Psi_{i, j-1 ; p, q}^{*}
\end{aligned}
$$

$$
\begin{aligned}
& \left\langle\tilde{x}_{i, j}, x_{i-1, j}\right\rangle= \\
& \sum_{q=1}^{j} \Theta_{i, j ; 0, q} W_{0, q} \Phi_{i-1, j ; 0, q}^{*}+\sum_{p=1}^{i-1} \Theta_{i, j ; p, 0} W_{p, 0} \Phi_{i-1, j ; p, 0}^{*} \\
& +\sum_{p=0}^{i-1} \sum_{q=0}^{j}\left(\Xi_{i, j ; p, q} Q_{p, q}+\Pi_{i, j ; p, q} S_{p, q}\right) \Psi_{i-1, j ; p, q}^{*}
\end{aligned}
$$

Now we need to compute the term $\left\langle\tilde{x}_{i, j}, B_{i, j-1}^{(1)} w_{i, j-1}+\right.$ $\left.B_{i-1, j}^{(2)} w_{i-1, j}\right\rangle$. By (1) and (7), we have

$$
\begin{aligned}
\tilde{x}_{i, j} & =x_{i, j}-\hat{x}_{i, j} \\
& =A_{i, j-1}^{(1)} \tilde{x}_{i, j-1}+A_{i-1, j}^{(2)} \tilde{x}_{i-1, j}+B_{i, j-1}^{(1)} w_{i, j-1} \\
& +B_{i-1, j}^{(1)} w_{i-1, j}-\left(\sum_{l=0}^{j-1} K_{i, j-1 ; l}^{(1)} e_{i, l}+\sum_{k=0}^{i-1} K_{i-1, j ; k}^{(2)} e_{k, j}\right)
\end{aligned}
$$

Since for $0 \leq l \leq j$ and $0 \leq k \leq i$ we have $w_{i, j-1} \perp \tilde{x}_{i, j-1}, w_{i, j-1} \perp \tilde{x}_{i-1, j}, w_{i, j-1} \perp e_{i, l}, w_{i, j-1} \perp e_{k, j}$, and $\quad w_{i-1, j} \perp \tilde{x}_{i, j-1}, \quad w_{i-1, j} \perp \tilde{x}_{i-1, j}, \quad w_{i-1, j} \perp e_{i, l}$, $w_{i-1, j} \perp e_{k, j}, w_{i, j-1} \perp w_{i-1, j}$, then we find

$$
\begin{aligned}
& \left\langle\tilde{x}_{i, j}, B_{i, j-1}^{(1)} w_{i, j-1}+B_{i-1, j}^{(2)} w_{i-1, j}\right\rangle \\
= & B_{i, j-1}^{(1)} Q_{i, j-1}\left(B_{i, j-1}^{(1)}\right)^{*}+B_{i-1, j}^{(2)} Q_{i-1, j}\left(B_{i-1, j}^{(2)}\right)^{*} .
\end{aligned}
$$

This completes the proof.

Based on Theorem 1 together with Theorem 2 and Theorem 3, an algorithm for the Kalman filtering of 2-D systems can be obtained.

\section{$\underline{\text { Algorithm }}$}

For $(p, q),(k, l) \in \Xi$, let $\hat{x}_{p, 0}=0, \hat{x}_{0, q}=0, e_{p, 0}=y_{p, 0}$, $e_{0, q}=y_{0, q}, P_{p, 0}=W_{p, 0}, P_{0, q}=W_{0, q}$. Matrices $\Phi_{p, 0 ; k, 0}$, $\Phi_{0, q ; 0, l}, \Psi_{p, 0 ; k, 0}, \Psi_{0, q ; 0, l}$ are given by (15), and $\Theta_{p, 0 ; k, 0}$, $\Theta_{0, q ; 0, l}, \Xi_{p, 0 ; k, 0}, \Xi_{0, q ; 0, l}, \Pi_{p, 0 ; k, 0}, \Pi_{0, q ; 0, l}$ are given by (22).

For $j=1: N_{2}$

for $i=1: N_{1}$

Compute $\Phi_{i, j ; 0, l}, \Phi_{i, j ; k, 0}$ and $\Psi_{i, j ; k, l}$ for $l \in\{0, \ldots, j\}$ and $k \in\{0, \ldots, i\}$, using (13);

Compute $\Theta_{i, j ; 0, l}, \Theta_{i, j ; k, 0}, \Xi_{i, j ; k, l}$ and $\Pi_{i, j ; k, l}$ for $l \in\{0, \ldots, j\}$ and $k \in\{0, \ldots, i\}$, using (19), (20) and (21);

Compute $K_{i, j ; l}^{(1)}$ and $K_{i, j ; k}^{(2)}$ for $l \in\{0, \ldots, j\}$ and $k \in\{0, \ldots, i\}$, by (24) and (25);

Compute $\hat{x}_{i, j}$ by (7) and then compute

$$
e_{i, j}=y_{i, j}-C_{i, j} \hat{x}_{i, j} \text {; }
$$

Compute $P_{i, j}$ using (27);

end

end 
In the next section, a simplified version of 2-D Kalman filter is provided which cannot achieve an optimal estimation but is easier to apply. The performance analysis of such simplified Kalman filter is also given.

\section{A SIMPLIFIED VERSION OF 2-D KALMAN FILTER}

Given $(i, j) \in \Omega$, for the 2-D system in the form of (1), we consider a simplified 2-D Kalman filter having the following structure

$$
\begin{aligned}
\hat{x}_{i+1, j+1}= & A_{i+1, \hat{x}_{i+1, j}}^{(1)} A_{i, j+1}^{(2)} \hat{x}_{i, j+1} \\
& +K_{i+1, j+1 ; j}^{(1)} e_{i+1, j}+K_{i+1, j+1 ; i}^{(2)} e_{i, j+1} \\
e_{i, j}= & y_{i, j}-C_{i, j} \hat{x}_{i, j}
\end{aligned}
$$

with boundary conditions $\hat{x}_{k, 0}=0$ and $\hat{x}_{0, l}=0$ for $k \leq i$ and $l \leq j$, respectively, where

$$
\begin{aligned}
& K_{i+1, j+1 ; j}^{(1)}= \\
& \left(A_{i, j+1}^{(2)}\left\langle x_{i, j+1}, \tilde{x}_{i+1, j}\right\rangle C_{i+1, j}^{*}+A_{i+1, j}^{(1)} P_{i+1, j} C_{i+1, j}^{*}\right) T_{i+1, j}^{-1} \\
& K_{i+1, j+1 ; i}^{(2)}= \\
& \left(A_{i+1, j}^{(1)}\left\langle x_{i+1, j}, \tilde{x}_{i, j+1}\right\rangle C_{i, j+1}^{*}+A_{i, j+1}^{(2)} P_{i, j+1} C_{i, j+1}^{*}\right) T_{i, j+1}^{-1}
\end{aligned}
$$

and

$$
\begin{aligned}
\left\langle x_{i, j+1}, \tilde{x}_{i+1, j}\right\rangle= & \sum_{p=1}^{i} \Phi_{i, j+1 ; p, 0} W_{p, 0} \Theta_{i+1, l ; p, 0}^{*} \\
& +\sum_{q=1}^{j} \Phi_{i, j+1 ; 0, q} W_{0, q} \Theta_{i+1, j ; 0, q}^{*} \\
& +\sum_{p=0}^{i} \sum_{q=0}^{j} \Psi_{i, j+1 ; p, q} Q_{p, q} \Xi_{i+1, j ; p, q}^{*} \\
& +\sum_{p=0}^{i} \sum_{q=0}^{j} \Psi_{i, j+1 ; p, q} S_{p, q} \Pi_{i+1, j ; p, q}^{*}, \\
\left\langle x_{i+1, j}, \tilde{x}_{i, j+1}\right\rangle= & \sum_{p=1}^{i} \Phi_{i+1, j ; p, 0} W_{p, 0} \Theta_{i, j+1 ; p, 0}^{*} \\
& +\sum_{q=1}^{j} \Phi_{i+1, j ; 0, q} W_{0, q} \Theta_{i, j+1 ; 0, q}^{*} \\
& +\sum_{p=0}^{i} \sum_{q=0}^{j} \Psi_{i+1, j ; p, q} Q_{p, q} \Xi_{i, j+1 ; p, q}^{*} \\
& +\sum_{p=0}^{i} \sum_{q=0}^{j} \Psi_{i+1, j ; p, q} S_{p, q} \Pi_{i, j+1 ; p, q}^{*}
\end{aligned}
$$

where matrices $\Phi_{i, j ; p, 0}$ and $\Phi_{i, j ; 0, q}, \Psi_{i, j ; p, q}$ are given in (13) and (14), and matrices $\Theta_{i, j ; 0, q}, \Theta_{i, j ; p, 0}, \Xi_{i, j ; p, q}$ and $\Pi_{i, j ; p, q}$ are given as follows

$$
\begin{aligned}
& \Theta_{i, j ; 0, q}=A_{i, j-1}^{(1)} \Theta_{i, j-1 ; 0, q}+A_{i-1, j}^{(2)} \Theta_{i-1, j ; 0, q} \\
& \quad-K_{i, j ; j-1}^{(1)} C_{i, j-1} \Theta_{i, j-1 ; 0, q}-K_{i, j ; i-1}^{(2)} C_{i-1, j} \Theta_{i-1, j ; 0, q} \\
& \Theta_{i, j ; p, 0}=A_{i, j-1}^{(1)} \Theta_{i, j-1 ; p, 0}+A_{i-1, j}^{(2)} \Theta_{i-1, j ; p, 0} \\
& \quad-K_{i, j ; j-1}^{(1)} C_{i, j-1} \Theta_{i, j-1 ; p, 0}-K_{i, j ; i-1}^{(2)} C_{i-1, j} \Theta_{i-1, j ; p, 0}
\end{aligned}
$$

$$
\begin{aligned}
& \Xi_{i, j ; p, q} \\
& =\left\{\begin{array}{lr}
0 & p=i, q=j, \\
B_{i, j-1}^{(1)} & p=i, q=j-1, \\
B_{i-1, j}^{(2)} & p=i-1, q=j, \\
\left(A_{i, j-1}^{(1)}-K_{i, j ; j-1}^{(1)} C_{i, j-1}\right) \Xi_{i, j-1 ; p, q} \\
+\left(A_{i-1, j}^{(2)}-K_{i, j ; i-1}^{(2)} C_{i-1, j}\right) \Xi_{i-1, j ; p, q} \\
\text { otherwise. }
\end{array}\right.
\end{aligned}
$$

$\Pi_{i, j ; p, q}$

$$
=\left\{\begin{array}{cr}
0, & p=i, q=j, \\
\left(A_{i-1, j}^{(2)}-K_{i, j ; i-1}^{(2)} C_{i-1, j}\right) \Pi_{i-1, j ; p, j}-K_{i, j ; p}^{(2)} \\
& q=j, p<i \\
\left(A_{i, j-1}^{(1)}-K_{i, j ; j-1}^{(1)} C_{i, j-1}\right) \Pi_{i, j-1 ; i, q}-K_{i, j ; q}^{(1)} & p=i, q<j, \\
& \\
\left(A_{i, j-1}^{(1)}-K_{i, j ; j-1}^{(1)} C_{i, j-1}\right) \Pi_{i, j-1 ; p, q} \\
+\left(A_{i-1, j}^{(2)}-K_{i, j ; i-1}^{(2)} C_{i-1, j}\right) \Pi_{i-1, j ; p, q} \\
p<i, q<j
\end{array}\right.
$$

The estimation error $P_{i, j}:=\left\langle\tilde{x}_{i, j}, \tilde{x}_{i, j}\right\rangle$ is expressed by (8) and $T_{i, j}:=C_{i, j} P_{i, j} C_{i, j}^{*}+R_{i, j}$.

This computation scheme is similar to the algorithm shown in Section III. The difference lies in the structure of the filter and the computation of matrices $\Theta_{i, j ; 0, q}, \Theta_{i, j ; p, 0}, \Xi_{i, j ; p, q}$ and $\Pi_{i, j ; p, q}$. Compared with the Kalman filter given in Section III, the advantage of this simplified filter is that the estimation $\hat{x}_{i, j}$ only depends on $\hat{x}_{i, j-1}, \hat{x}_{i-1, j}, e_{i, j-1}$ and $e_{i-1, j}$ rather than $e_{i, l}$ and $e_{k, j}$ with $l \in\{0, \ldots, j\}, k \in\{0, \ldots, i\}$. Therefore, less memory is needed for the computation of the simplified Kalman filter.

\section{REFERENCES}

[1] C. Du, L. Xie and Y.C. Soh, $H_{\infty}$ filtering of 2-D discrete systems, IEEE Transactions on Signal Processing, Vol. 48, No. 6, pp. 17601768, 2000.

[2] C. Du and L. Xie, $H_{\infty}$ Control and Filtering of Two-dimensional Systems, Springer, 2002.

[3] E. Fornasini and G. Marchesini, State-space realization theory of twodimensional filters. IEEE Transactions on Automatic Control, Vol.21, pp. 484-491, 1976

[4] H. Gao, J. Lam, C. Wang and S. Xu, Robust $H_{\infty}$ filtering for 2D stochastic systems, Circuits, Systems and Signal Processing, Vol.23, No. 6, pp. 479-505, 2004.

[5] A. Habibi, Two-Dimensional Bayesian estimation of image, Proceedings of the IEEE, Vol. 60, pp. 878-883, 1972.

[6] T. Kailath, A.H. Sayed and B. Hassibi, Linear Estimation, Prentice Hall, 2000.

[7] R.E. Kalman, A New Approach to Linear Filtering and Prediction Problems,Transaction of the ASME - Journal of Basic Engineering, Vol. 82, pp. 35-45, March 1960

[8] T. Katayama and M. Kosaka, Recursive filtering algorithm for a 2-D system, IEEE Transactions on Automatic Control, Vol.24, No. 1, pp. 130-132, 1979.

[9] C. Li and M.S. Fadali, Optimal control of 2-D systems, IEEE Transactions on Automatic Control, Vol.36, pp. 223-228, 1991.

[10] W.S. Lu, On a Lyaponov approach to stability analysis of 2-D digital filters, IEEE Trans. on Circuits and Systems, I, Vol.41, pp. 665-669, 1993.

[11] W.A. Porter and J.L. Aravena, State estimation in discrete m-D systems, IEEE Transactions on Automatic Control, Vol. 31, No. 3, pp. 280-283, 1986 
[12] R.P. Roesser, A discrete state-space model for linear image processing. IEEE Transactions on Automatic Control, Vol.20, pp. 1-10, 1975.

[13] M. Śebek, Polynomial solution of 2-D Kalman-Bucy filtering problems, IEEE Transactions on Automatic Control, Vol. 37, No. 10, pp. 1530-1533, 1992.

[14] H.D. Tuan, P. Apkarian, T.Q. Nguyen and T. Narikiyo, Robust mixed $\mathrm{H}_{2} / \mathrm{H}_{\infty}$ filtering of 2-D systems. IEEE Transactions on Signal Processing, Vol. 50, No. 7, pp. $1759-1771,2002$.

[15] J.W. Woods and C.H. Radewan, Kalman filtering in two dimensions, IEEE Transactions on Information Theory, Vol.23, No. 4, pp. 473-482, 1977.

[16] J.W. Woods and V.K. Ingel, Kalman filtering in two dimensions: further results, IEEE Transactions on Acoustics, Speech, and Signal Processing, Vol. 29, No. 2, pp. 188-197, 1981.

[17] R. Yang, L. Xie and C. Zhang, Generalized two-dimensional KalmanYakubovich-Popov lemma for discrete Roesser model, IEEE Transactions on Circuits and Systems: -I, Vol. 55, No. 10, pp. 3223-3233, 2008.

[18] R. Yang, L. Xie and C. Zhang, $\mathrm{H}_{2}$ and Robust $\mathrm{H}_{2} / \mathrm{H}_{\infty}$ Control of 2-Dimensional Systems in Roesser Model, Automatica, Vol.42, No. 9, pp. 1507-1514, 2006.

[19] Y. Zou, M. Sheng, N. Zhong and S. Xu, A generalized Kalman Filter for 2D discrete systems, Circuits, Systems and Signal Processing, Vol. 23, No. 5, pp.351-364, 2004. 